\title{
Ethical dilemmas in global anesthesia and surgery
}

\author{
Gregory Klar, MD, FRCPC, MIPH@ - Julie Zalan, MD, FRCPC • Anthony M. Roche, MBChB, FRCA • \\ Rachel Phelan, MSc
}

Received: 18 October 2017/Revised: 20 March 2018/Accepted: 21 March 2018/Published online: 14 May 2018

(c) Canadian Anesthesiologists' Society 2018

Historically, surgical, anesthetic, and perioperative services have been neglected in low- and middle-income countries (LMICs) by many, including global health funding organizations and major donors. ${ }^{1}$ Several ethical dilemmas surround this neglect, and this editorial aims to explain why allowing the perpetuation of this neglect in LMICs represents an ethically unacceptable tenet. Indeed, we argue that it is unconscionable for the global community to wait for a solution to spontaneously evolve. Rather, we need to become engaged to formulate sustainable solutions.

The Global Burden of Disease (GBD) project continues to provide emerging data highlighting the extent of the surgical disease burden. This burden is defined as disability and premature death that exist in a population without surgical care that could be averted if surgical care were readily available. ${ }^{2}$ The global surgical burden is estimated to be $28-32 \%$ of the total GBD and affects mostly LMICs. $^{2,3}$ To put things in perspective, this is greater than the global burden of HIV, tuberculosis, and malaria combined. ${ }^{2}$ Major global health players including the

G. Klar, MD, FRCPC, MIPH · J. Zalan, MD, FRCPC

R. Phelan, MSc

Department of Anesthesiology and Perioperative Medicine,

Queen's University, Kingston, ON, Canada

G. Klar, MD, FRCPC, MIPH ( $\square)$

Department of Anesthesiology and Perioperative Medicine, Queen's University, Victory 2, Kingston Health Sciences Centre, Kingston General Hospital Site, 76 Stuart Street, Kingston, ON

K7L 2V7, Canada

e-mail: gklar@rcsi.ie

A. M. Roche, MBChB, FRCA

Department of Anesthesiology, University of Washington,

Seattle, WA, USA
World Health Organization, ${ }^{1}$ Lancet Commission on Global Surgery (LCoGS), ${ }^{4}$ and Disease Control Priorities $^{5}$ have recently highlighted the global surgical inequities; this recognition represents significant progress in itself. $^{6}$ There are also increasing numbers of nongovernmental organizations and university partnerships that provide surgical aid. Among Canadian anesthesiologists, there is an increasing awareness and interest in international aid shown by increasing involvement in international health projects. ${ }^{7}$ This is, in part, because of advocacy by organizations such as LCoGS and the Canadian Anesthesiologists' Society International Education Foundation. Nevertheless, evidence for progress using standardized metrics of decreased global surgical morbidity and mortality remains forthcoming.

\section{Are surgical inequities a new concept?}

After the creation of The United Nations in 1945, largescale international aid efforts became more prevalent to battle global health inequalities. Throughout the 1960s and 1970s, increasing aid efforts were directed from the geographical north to the geographical south. Essential surgical care and primary healthcare were neglected and continued to be neglected for decades. In an attempt to rectify the disproportionate emphasis on infectious disease, a landmark meeting was held in 1978 in Alma-Ata, USSR. The closing statement emphasized that primary healthcare services (including surgery) should be available to all by 2000. Nevertheless, infectious disease remained center stage, and the outbreak of human immunodeficiency virus/ acquired immunodeficiency syndrome only further distracted the donor and aid community. 


\section{Is surgery a cost-effective global health priority?}

It was only near the end of the Millennium Development Goal era ${ }^{8}$ that the global health community started to focus more attention on the surgical inequities. With this increased attention, common historical misconceptions, which had previously contributed as major barriers to achieving surgical equality in LMICs, were dispelled. In particular, and contrary to previous beliefs, surgery was proven to be cost-effective compared with other health interventions. ${ }^{9}$ Comprehensive surgical support not only reduces morbidity and mortality but also increases productivity and supports healthier economies. In a statement to the LCoGS, the President of The World Bank, Dr. Jim Kim, reiterated the important link between the global burden of surgical disease and economic growth. ${ }^{10}$ The current plans of the LCoGS to ramp up surgical services to meet $80 \%$ of the global surgical demand are costly. Nevertheless, the LCoGS estimates that by ignoring this demand, LMICs stand to lose 12.3 trillion dollars between 2015 and 2030 as a result of decreased economic productivity - a full 35 times the cost of scaling up surgical services. ${ }^{3}$ We now know that conditions amenable to surgical intervention disproportionately affect LMICs and make up $28-32 \%$ of the GBD. ${ }^{3}$ The global recognition that surgical conditions are a high priority in LMICs was shown by the development and adoption of the World Health Assembly (WHA) 68.15 for "Strengthening emergency and essential surgical care and anesthesia as a component of universal health coverage" at their $68^{\text {th }}$ meeting in 2015-something the WHA remains committed to. ${ }^{11,12}$ We do not suggest that the ramping of surgical services should be done at the expense of other global health priorities such as other non-communicable disease and communicable diseases. Rather we argue that there is a need for a proportionate response to the global surgical inequities, a response that has not been put forth by major donors. ${ }^{13}$

\section{Is access to surgery a human right?}

In 1948, The Declaration of Human Rights addressed aspects of liberties to which a person in the world is entitled. ${ }^{14}$ Within the declaration, article 25 specifically defines health as a human right stating that,

"Everyone has the Right to a standard of living adequate for the health and well-being of himself and of his family, including...medical care and necessary social services...". ${ }^{14}$

In 1966, the International Covenant on Economic, Social, and Cultural Rights ${ }^{15}$ re-iterated that every person "has the right to the highest attainable standard of physical and mental health". Although this declaration does not specify the birth environment, it does state that "core" healthcare necessities are a human right. ${ }^{15}$ Furthermore, surgery has been accepted by the WHO, The World Bank, and the LCoGS as an "indivisible and indispensable part of health care". ${ }^{3}$ Since access to surgery is uniformly acknowledged as a human right, it is clear that the public health world and medical/paramedical professions have an ethical obligation to provide solutions to eliminate global surgical inequities.

Are surgical inequities ethically disconcerting?

We have established that surgical services are part of primary healthcare and therefore a basic human right. We also know that surgical services are cost-effective interventions that are high priority for LMICs. Should we not therefore question why there has not been more accomplished in the establishment of sustainable surgical services in LMICs? One's birthplace, at least temporarily (and often permanently), defines the level of surgical care. One's access to health and ability to function should not be left to chance. Yet, only $3.5 \%$ of the 234 million surgical procedures performed annually worldwide are undertaken in LMICs. ${ }^{3}$ The equipment and support frequently do not meet the standards of the World Federation of Societies of Anaesthesiologists put forth in 1992 and revised in 2010 and 2018. ${ }^{16}$ In fact, these standards have not been achieved in many parts of the world. ${ }^{17}$

\section{Position of prominent ethicists on global health inequities}

Prominent public health ethicists Thomas Nagel and John Rawl agree that health inequities are morally unacceptable. ${ }^{18}$ This is further supported by Norman Daniel, an American philosopher and ethicist, who developed the idea of justice in health. In his book, Just Health, he explores this concept and speaks to the effect that healthcare needs to be distributed fairly across social, demographic, and geographic barriers. ${ }^{19} \mathrm{He}$ supports policy-makers and urges them to assist populations in the need for decreasing health inequities. Daniel's philosophy was further explored by two human rights lawyers, Ooms and Hammonds. ${ }^{20}$ While the two lawyers did agree with Daniel, they took the rhetoric even further and highlighted the fact that global health inequities equally have no moral standing as we live in a global state. They concluded that there is a global responsibility and obligation to minimize inequalities and this transcends national borders. ${ }^{20}$ 
Ethically questionable aid-is it applicable to anesthesia?

A significant amount of literature and press has questioned the effectiveness of aid in LMICs. Moyo, a leading economist, popularized the idea that aid, in most circumstances, does not work and causes harm because it leads to aid dependence (and loss of independence), ${ }^{21}$ a view not reciprocated by many including other leading economists such as Sachs. ${ }^{22}$ Surgical international aid projects including teaching partnerships are often criticized as lacking impact, sustainability, and self-evaluation. Furthermore, most current aid projects are not based on "evidence" but rather anecdotal experience and individual opinion. Issues of transparency, accountability, governance, and cultural appropriateness also arise.

As we transition into an era of evidence-based public health, surgical programs should strive towards providing educational and partnership assistance based upon evidence, audits, and good governance of the programs in LMICs. Current recommendations in the international anesthesia health literature include the creation of lasting partnerships with harmonized efforts that fit within local geopolitical environments.

\section{Is "brain drain" an ethical concern?}

Further compounding the issue of surgical inequity is the loss of trained professionals to high-income countries. The International Organization of Migration estimates that LMICs spend $\$ 500$ million each year to educate health workers who then leave to work abroad. ${ }^{23}$ Clearly this was not the intention of the supporting host countries. This introduces an ethical dilemma in its own right because nations with the greatest need are left with the least support. Of all the world's healthcare workers, $37 \%$ live in North America, which carries only $10 \%$ of the GBD. ${ }^{23}$ In contrast, as of 2006, Africa carried $24 \%$ of the GBD with only $3 \%$ of the world's healthcare workers. ${ }^{24}$

\section{Conclusion}

Although health and access to healthy living are human rights that should not be determined by one's geographical environment, this is the reality for over 5 billion people who lack access to safe and affordable surgical care. ${ }^{3}$ There is a critical need for international collaborations and solutions focused upon decreasing the unacceptable global surgical burden. Because each region is different, local involvement and locally driven initiatives are essential to establishing the necessary infrastructure and developing locally relevant solutions. Creating partnerships and providing evidence-based interventions focusing upon capacity-building are essential for achieving this goal. While international donors are contributing to remedying the global surgical inequities, the current level of aid remains insufficient ${ }^{13}$ even though estimated costs are small relative to the economic and welfare returns on investment. ${ }^{3}$ Financial incentives and/or terms need to be established and implemented in an attempt to minimize brain drain. Anesthesiology must play a major role in advocating for safe surgery and anesthesia on a global scale, which, after all, is a basic human right.

\section{Dilemmes éthiques en anesthésie et chirurgie à l'échelle internationale}

Historiquement, les services chirurgicaux, anesthésiques et périopératoires ont été négligés dans les pays à revenus faibles et intermédiaires (PRFI) par beaucoup, y compris les organismes internationaux de financement de la santé et les principaux donateurs. ${ }^{1}$ Un certain nombre de dilemmes éthiques entourent ce manque d'attention et cet éditorial a pour but d'expliquer pourquoi la persistance de cette négligence dans les PRFI représente une doctrine éthiquement inacceptable. En fait, il nous paraît scandaleux que la communauté internationale attende qu'une solution spontanée apparaisse. Nous devrions plutôt nous engager à formuler des solutions durables.

Le projet sur la charge mondiale de morbidité (CMM) continue de fournir des données nouvelles qui soulignent l'étendue du fardeau des maladies chirurgicales. Cette morbidité est définie par l'invalidité et les décès prématurés frappant une population dénuée de soins chirurgicaux et qui pourraient avoir été évités si de tels soins avaient été disponibles. $^{2}$ On estime que la charge chirurgicale mondiale représente $28 \%$ à $32 \%$ de la CMM totale et concerne principalement les PRFI.,3 Pour remettre les choses en perspective, cela représente plus que le fardeau global du VIH, de la tuberculose et du paludisme combinés. $^{2}$ Les principaux acteurs internationaux du domaine de la santé, dont l'Organisation mondiale de la santé, ${ }^{1}$ la Lancet Commission on Global Surgery (LCoGS), ${ }^{4}$ et Disease Control Priorities ${ }^{5}$ ont récemment souligné les inégalités en matière de chirurgie dans le monde; en soi, cette reconnaissance représente un progrès significatif. $^{6}$ Il existe également un nombre croissant d'organisations non gouvernementales (ONG) et de partenariats avec des universités qui apportent une aide chirurgicale. Parmi les anesthésiologistes canadiens, on constate une sensibilisation et un intérêt croissants pour l'aide internationale comme le prouve une plus grande 
implication dans des projets de santé internationaux. ${ }^{7}$ Ceci est en partie dû à l'action militante d'organismes comme la LCoGS et la Fondation d'éducation internationale de la Société canadienne des anesthésiologistes. Néanmoins, il reste encore à voir les preuves des progrès accomplis à partir des paramètres standardisés de baisse de morbidité et mortalité chirurgicales mondiales.

\section{Les inégalités chirurgicales sont-elles un nouveau concept?}

Après la création des Nations-Unies en 1945, des efforts internationaux d'aide à grande échelle sont devenus plus fréquents pour lutter contre les inégalités de santé dans le monde. Tout au long des années 1960 et 1970, des efforts d'aide croissants ont été dirigés du Nord vers le Sud de la planète. Les soins chirurgicaux essentiels et les soins de santé primaires ont toutefois été négligés et ont continué à l'être pendant des décennies. Dans un effort pour rectifier l'importance disproportionnée accordée aux maladies infectieuses, une réunion capitale s'est tenue en 1978 à Alma-Ata, alors dans l'ancienne Union soviétique. La déclaration finale de la conférence a insisté sur le fait que les services de santé primaires (y compris chirurgicaux) devraient être accessibles à tous avant l'an 2000. Les maladies infectieuses sont tout de même restées au centre de la scène et l'épidémie de virus de l'immunodéficience humaine/syndrome d'immunodéficience acquise n'a fait que détourner davantage l'attention des donneurs et de la communauté des organismes d'aide internationale.

\section{La chirurgie est-elle une priorité de santé rentable?}

Ce n'est que vers la fin de l'ère des Buts de développement du millénaire ${ }^{8}$ que la communauté médicale internationale a commencé à porter davantage d'attention aux inégalités chirurgicales. Avec l'accroissement de cet intérêt, des idées historiques erronées fréquentes - qui avaient précédemment constitué des obstacles majeurs à l'atteinte de l'égalité chirurgicale dans les PRFI — ont été corrigées. Notamment, et en contradiction avec des croyances antérieures, la chirurgie a démontré un rapport coût-efficacité favorable par rapport aux autres interventions de santé. ${ }^{9}$ Non seulement un soutien chirurgical complet réduit la morbidité et la mortalité, mais il augmente également la productivité et renforce la santé des économies. Dans une déclaration de la LCoGS, le président de la Banque mondiale, M. Jim Kim, a réitéré le lien important existant entre la charge mondiale des maladies chirurgicales et la croissance économique. ${ }^{10}$ Les plans actuels de la LCoGS pour renforcer les services chirurgicaux afin de répondre à $80 \%$ de la demande chirurgicale internationale sont onéreux. Mais la LCoGS estime qu'en ignorant cette demande, les PRFI perdront 12,3 milliards de dollars entre 2015 et 2030 du fait d'une moindre productivité économique, soit 35 fois le coût de la mise à l'échelle des services chirurgicaux. ${ }^{3}$ Nous savons aujourd'hui que les pathologies pouvant être guéries par une intervention chirurgicale affectent de manière disproportionnée les PRFI et représentent jusqu'à $28 \%$ à $32 \%$ de la $\mathrm{CMM}^{3}{ }^{3}$ La reconnaissance internationale de la grande priorité que représentent les maladies chirurgicales dans les PRFI a été montrée par l'élaboration et l'adoption par l'Assemblée mondiale de la santé (WHA) 68.15 de la déclaration suivante lors de sa $68^{\mathrm{e}}$ conférence en 2015 : «Le renforcement des soins chirurgicaux et de l'anesthésie, urgents et essentiels [sont] un élément de la couverture maladie universelle »; une action envers laquelle la WHA continue de s'engager. ${ }^{11,12}$ Nous ne suggérons pas que l'accroissement des services chirurgicaux doive se faire aux dépens des autres priorités de santé globales, telles que les autres maladies non transmissibles et transmissibles. Nous défendons plutôt l'idée qu'une action proportionnée est nécessaire pour répondre aux inégalités chirurgicales mondiales, une réponse qui n'a pas été avancée par les principaux donateurs. ${ }^{13}$

\section{L'accès à la chirurgie est-il un droit humain?}

En 1948, la Déclaration des droits de la personne a abordé la question des libertés auxquelles une personne a droit dans le monde. ${ }^{14}$ Dans la déclaration, l'article 25 de façon spécifique définit spécifiquement la santé comme un droit de l'homme, indiquant que

« Toute personne a droit à un niveau de vie suffisant pour assurer sa santé, son bien-être et ceux de sa famille, notamment pour [...] les soins médicaux ainsi que pour les services sociaux nécessaires... ». ${ }^{14}$

En 1966, le Pacte international relatif aux droits économiques, sociaux et culturels ${ }^{15}$ a rappelé que « toute personne [a le droit] de jouir du meilleur état de santé physique et mentale qu'elle soit capable d'atteindre ». Même si cette déclaration ne précise pas l'environnement de la naissance, elle déclare que les nécessités «fondamentales » pour la santé sont un droit humain. ${ }^{15}$ De plus, la chirurgie a été reconnue par L'OMS, la Banque mondiale et la LCoGS comme une "partie indivisible et indispensable des soins de santé »..$^{3}$ Dans la mesure où l'accès à la chirurgie est uniformément reconnu comme un droit humain, il est clair que le monde de la santé publique et les professions paramédicales ont l'obligation éthique de 
fournir des solutions pour éliminer les inégalités chirurgicales dans le monde.

Les inégalités chirurgicales sont-elles éthiquement déconcertantes?

Nous avons établi que les services chirurgicaux font partie des soins de santé primaires et sont donc un droit humain fondamental. Nous savons également que les services chirurgicaux sont des interventions rentables et sont une grande priorité pour les PRFI. Ne doit-on pas, dès lors, se demander pourquoi il n'a pas été fait plus pour développer des services chirurgicaux durables dans les PRFI? Le lieu de naissance d'une personne définit, au moins temporairement (et souvent de manière permanente), le niveau des soins chirurgicaux. L'accès d'une personne à la santé et son aptitude à fonctionner normalement ne devraient pas être laissés au hasard. Cependant, seulement $3,5 \%$ des 234 millions de procédures chirurgicales pratiquées chaque année dans le monde le sont dans les PRFI. ${ }^{3}$ Souvent, l'équipement et le soutien ne répondent pas aux normes établies par la Fédération mondiale des sociétés d'anesthésiologie en 1992 et révisées en $2010 .{ }^{16}$ En fait, ces normes n'ont jamais été atteintes dans de nombreuses parties du monde. ${ }^{17}$

\section{L'avis d'éthiciens de renom sur les inégalités mondiales en matière de santé}

Thomas Nagel et John Rawl, des éthiciens de renom en matière de santé publique, sont d'accord pour dire que les inégalités en santé sont moralement inacceptables. ${ }^{18} \mathrm{Ils}$ sont également soutenus par Normal Daniels, philosophe et éthicien américain, qui a élaboré l'idée de justice dans la santé. Dans son livre, Just Health, ce dernier explore le concept et parle de l'effet que les besoins en soins de santé ont d'être justement distribués indépendamment des obstacles sociaux, démographiques et géographiques. ${ }^{19}$ Il soutient les décideurs politiques et les presse d'aider les populations dans leur besoin de diminuer les inégalités en matière de santé. La philosophie de Daniels a aussi été explorée par deux avocats des droits humains, Ooms et Hammonds. ${ }^{20}$ Tandis que les deux avocats étaient d'accord avec Daniels, ils ont poussé la rhétorique encore plus loin et souligné le fait que les inégalités de santé dans le monde n'ont pas de fondement moral, car nous vivons dans un état global. Ils ont conclu qu'il existe une responsabilité et une obligation internationales envers la réduction des inégalités et que cela transcende les frontières nationales. ${ }^{20}$
Aide éthiquement douteuse : cela s'applique-t-il à l'anesthésie?

Une quantité significative de documents et d'articles de presse a mis en cause l'efficacité de l'aide dans les PRFI. Mme D. Moyo, spécialiste de la macroéconomie, a popularisé l'idée que l'aide ne fonctionne pas dans la majorité des cas et s'avère nocive, car menant à une dépendance envers cette aide (et la perte de l'indépendance), ${ }^{21}$ un point de vue qui n'est pas partagé par de nombreux autres économistes de renom tel que Sachs. $^{22}$ Les projets internationaux d'aide chirurgicale incluant des partenariats sont souvent critiqués pour manquer de répercussions, de durabilité et d'auto-évaluation. En outre, les projets d'aide les plus récents ne reposent pas sur des «données probantes », mais plutôt sur des expériences anecdotiques et des opinions personnelles. Les enjeux de la transparence, de la responsabilité envers les autres, de la gouvernance et de l'appropriation intellectuelle sont également soulevés.

En passant à une ère de santé publique basée sur des données probantes, les programmes chirurgicaux doivent s'efforcer d'aller vers une aide à la formation et au partenariat basée sur des preuves, des vérifications et une bonne gouvernance de ces programmes dans les PRFI. Les recommandations actuelles figurant dans les publications internationales sur la santé anesthésique incluent la création de partenariats durables avec des efforts harmonisés s'insérant dans des environnements géopolitiques locaux.

\section{« L'exode des cerveaux » est-il une préoccupation éthique?}

La perte de professionnels formés au profit des pays à revenus élevés vient encore aggraver le problème de l'inégalité chirurgicale. L'organisation internationale pour les migrations (OIM) estime que les PRFI dépensent annuellement 500 millions de dollars pour former des professionnels de la santé qui partent ensuite travailler à l'étranger. ${ }^{23}$ À l'évidence, cela n'était pas l'intention des pays hôtes qui les soutiennent. Cela introduit en soi un dilemme éthique dans la mesure où les nations en ayant le plus grand besoin sont laissées avec le moins de soutien. De tous les professionnels de la santé dans le monde, $37 \%$ vivent en Amérique du Nord qui ne supporte que $10 \%$ de la CMM. $^{23}$ En revanche, en 2006, l'Afrique supportait $24 \%$ de la CMM avec seulement $3 \%$ des employés du domaine de la santé dans le monde. ${ }^{24}$ 


\section{Conclusion}

Même si la santé et l'accès à une vie en bonne santé sont des droits humains qui ne devraient pas être déterminés par l'environnement géographique de chaque individu, cinq milliards de personnes vivent la réalité d'une absence d'accès à des soins chirurgicaux sécuritaires et abordables. ${ }^{3}$ Il y a un besoin criant de collaborations internationales et de solutions axées sur la diminution de la charge mondiale de morbidité chirurgicale globale inacceptable. Dans la mesure où chaque région est différente, une implication locale et des initiatives menées localement sont essentielles pour créer les infrastructures nécessaires et développer des solutions localement pertinentes. La création de partenariats et l'offre d'interventions reposant sur des données probantes et axées sur le renforcement des capacités autonomes sont indispensables pour atteindre cet objectif. Tandis que les donateurs internationaux contribuent à soulager les inégalités chirurgicales dans le monde, le niveau actuel de l'aide reste insuffisant ${ }^{13}$ même si les coûts estimés sont relativement faibles comparativement au rendement des investissements pour l'économie et le bien-être des populations. ${ }^{3}$ Des incitations économiques et/ou des modalités doivent être élaborées et mises en œuvre pour tenter de minimiser l'exode des compétences. L'anesthésiologie doit jouer un rôle majeur dans la défense d'une chirurgie et d'une anesthésie sécuritaires à grande échelle, ce qui — après tout — est un droit humain fondamental.

Conflicts of interest None of the authors have any real or perceived conflicts of interest associated with publication of the current manuscript.

Editorial responsibility This submission was handled by Dr. Hilary P. Grocott, Editor-in-Chief, Canadian Journal of Anesthesia.

Funding No funding was obtained for preparation of the current manuscript.

Conflits d'intérêts Aucun auteur n'a de conflits d'intérêts réels ou perçus associés à la publication de ce manuscrit.

Responsabilité éditoriale Cet article a été traité par le Dr Hilary P. Grocott, Rédacteur en chef, Journal canadien d'anesthésie.

Financement Aucun financement n'a été obtenu pour la préparation de ce manuscrit.

\section{References}

1. Yung Bae J, Groen RS, Kushner AL. Surgery as a public health intervention: common misconceptions versus the truth. Bulletin of the World Health Organization 2011; 89: 394. Available from
URL: http://www.who.int/bulletin/volumes/89/6/11-088229/en/ (accessed March 2018).

2. Bowman $K G$, Jovic $G$, Rangel $S$, Berry WR, Gawande AA. Pediatric emergency and essential surgical care in Zambian hospitals: a nationwide study. J Ped Surg 2013; 48: 1363-70.

3. Meara JG, Leather AJ, Hogander L, et al. Global Surgery 2030: evidence and solutions for achieving health, welfare, and economic development. Lancet 2015; 386: 569-624.

4. The Lancet Commission on Global Surgery. Executive Summary. Published April 28, 2015. Available from URL: http://www. thelancet.com/commissions/global-surgery (accessed March 2018).

5. Jamison DT, Breman JG, Measham AR, et al. Disease Control Priorities in Developing Countries, 2nd edition. Washington (DC): The International Bank for Reconstruction and Development / The World Bank; NY: Oxford University Press; 2006.

6. Soreide K, Alderson D, Bergenfelz A, et al. Strategies to improve clinical research in surgery thorugh international collaboration. Lancet 2013; 383: 1140-51.

7. Dumbarton TC, Bould MD. Thinking globally, training locally. Can J Anesth 2016; 63: 652-7.

8. United Nations. The Millenium Development Goals Report 2015. Available from URL: http://www.un.org/millenniumgoals/2015 MDG_Report/pdf/MDG\%202015\%20rev\%20(July\%201).pdf (accessed March 2018).

9. Grimes CE, Henry JA, Maraka J, Mkandawire NC, Cotton M. Cost-effectiveness of surgery in low- and middle-income countries: a systematic review. World J Surg 2014; 38: 252-63.

10. The Lancet Commission on Global Surgery. Dr Jim Kim: Address to The Lancet Commission on Global Surgery. 2015. Available from URL: http://www.lancetglobalsurgery.org/ (accessed March 2018).

11. World Health Assembly. Strengthening emergency and essential surgical care and anaesthesia as a component of universal health coverage. Sixty-eighth World Health Assembly. WHA 68.15 - 26 May 2015 Available from URL: http://apps.who.int/gb/ebwha/ pdf_files/WHA68/A68_R15-en.pdf (accessed: March 2018).

12. World Health Organization. Letter of commitment from WHO Director-General. Tedros: A healthier world - 21 April 2017. Available from URL: http://www.who.int/surgery/DrTedros Letter.pdf?ua=1 (accessed March 2018).

13. Gutnik LA, Dielman J, Dare AJ, et al. Funding flows to global surgery: an analysis of contributions from the USA. Lancet 2015 27; 385(Suppl 2): S51.

14. United Nations. Universal Declaration of Human Rights - 2015. Available from URL: http://www.un.org/en/udhrbook/pdf/udhr_ booklet_en_web.pdf (accessed March 2018).

15. Office of the High Commisioner for Human Rights. International Covenant on Economic, Social and Cultural Rights - 3 January 1976, in accordance with article 27. Available from URL: http:// www.ohchr.org/Documents/ProfessionalInterest/cescr.pdf (accessed March 2018).

16. Merry AF, Cooper JB, Soyannwo O, Wilson IH, Eichhorn JH. International Standards for a Safe Practice of Anesthesia 2010. Can J Anesth 2010; 57: 1027-34.

17. Enright A. Review article: safety aspects of anesthesia in underresourced locations. Can J Anesth 2013; 60: 152-8.

18. Ruger JP. Ethics and governance of global health inequalities. $\mathrm{J}$ Epidemiol Community Health 2006; 60: 998-1003.

19. Daniels N. Just Health: Meeting Health Needs Fairly. 1st ed. New York, NY: Cambridge University Press; 2008.

20. Ooms $G$, Hammonds $R$. Taking up Daniels' challenge: the case for global health justice. Health Hum Rights 2010; 12: 29-46.

21. Moyo D. Dead Aid. 1st ed. NY: Niall Ferguson; 2009 . 
22. Sachs J, McArthur JW. Moyo's confused attack on aid for Africa. Huffington Post. May 27, 2009 Available from URL: http:// probeinternational.org/library/wp-content/uploads/2011/12/JeffreySachs_-Moyos-Confused-Attack-on-Aid-for-Africa.pdf (accessed (March 2018).

23. Kuehn BM. Global shortage of health workers, brain drain stress developing countries. JAMA 2007; 298: 1853-5.
24. World Health Organization. The World Health Report 2006 working together for health. Available from URL: http://www. who.int/whr/2006/en/ (accessed March 2018). 